\title{
Detection of low-frequency earthquakes by matched filter technique using the product of mutual information and correlation coefficient
}

Ryo Kurihara ( $\nabla$ rkuri@eri.u-tokyo.ac.jp )

The University of Tokyo: Tokyo Daigaku https://orcid.org/0000-0003-2954-8486

Aitaro Kato

The University of Tokyo: Tokyo Daigaku

\section{Sumito Kurata}

The University of Tokyo: Tokyo Daigaku

Hiromichi Nagao

The University of Tokyo: Tokyo Daigaku

Full paper

Keywords: Matched filter technique, Low-frequency earthquakes, Mutual information, Kirishima volcano

Posted Date: August 5th, 2021

DOI: https://doi.org/10.21203/rs.3.rs-769134/v1

License: (9) This work is licensed under a Creative Commons Attribution 4.0 International License.

Read Full License

Version of Record: A version of this preprint was published at Earth, Planets and Space on December 1st, 2021. See the published version at https://doi.org/10.1186/s40623-021-01534-w. 


\section{Title: Detection of low-frequency earthquakes by matched}

\section{2 filter technique using the product of mutual information}

\section{3 and correlation coefficient}

4

5 Author \#1: Ryo Kurihara, Earthquake Research Institute, the University of Tokyo,

6 rkuri@eri.u-tokyo.ac.jp

7 Author \#2: Aitaro Kato, Earthquake Research Institute, the University of Tokyo,

8 akato@eri.u-tokyo.ac.jp

9 Author \#3: Sumito Kurata, Graduate School of Information Science and Technology,

10 the University of Tokyo, kurata@mist.i.u-tokyo.ac.jp

11 Author \#4: Hiromichi Nagao, Earthquake Research Institute, the University of Tokyo,

12 nagaoh@eri.u-tokyo.ac.jp

13

14 Indicate the corresponding author: Ryo Kurihara 
Matched filter technique is often used to detect microearthquakes such as

18 deep low-frequency (DLF) earthquakes. It compares correlation coefficients (CC)

19 between waveforms of template earthquakes and the observed data. Conventionally, the

20 sum of CC at multiple seismic stations is used as an index to detect the DLF

21 earthquakes. A major disadvantage of conventional method is drastically reduced

22 detection accuracy when there are too few seismic stations. A new matched filter

23 method proposed in this study can accurately detect microearthquakes using only a

24 single station. It adopts mutual information (MI) in addition to $\mathrm{CC}$ to measure the similarity between the template and target waveforms. The method uses the product of MI and CC (MICC) as an index to detect DLF earthquakes. This index shows a distinct peak corresponding to an earthquake signal in a synthetic data set consisting of artificial noise and the waveform of a DLF earthquake.

Application of this single-station method to field observations of Kirishima volcano, one of the most active volcanoes in Japan, detected a total of 354 DLF earthquakes from the data in December 2010, whereas the catalog of the Japan Meteorological Agency shows only two. The catalog of DLF earthquakes constructed here shows similar temporal behavior to that found by conventional matched filter method using the sum of the $\mathrm{CC}$ of the six stations near the volcano. The proposed method successfully identified approximately $80 \%$ of the earthquakes in the conventionally constructed catalogs. These results suggest that the proposed method can greatly contribute to the accurate cataloging of DLF earthquakes using only a single

38 seismic station.

Keywords

42 Matched filter technique, Low-frequency earthquakes, Mutual information, Kirishima 43 volcano 


\section{Introduction}

One of established methods for the automatic detection of microearthquakes, is the matched filter technique employing Pearson's correlation coefficients (CC) between template waveforms - which are previously seismic waveforms - and target waveforms. It can detect microearthquakes such as afterquakes (Peng and Zhao 2009), low-frequency earthquakes and low-frequency tremor in the plate subduction zone and volcanic regions (Gibbons and Ringdal 2006; Shelly et al. 2007; Shapiro et al. 2017; Yukutake et al. 2019; Kurihara et al. 2019; Kato and Nakagawa 2020). The matched filter method is especially useful in situations of continued intense seismic activity such as aftershocks and seismic swarms because the waveforms of low-magnitude earthquakes are masked by the large-amplitude waves from multiple events. The method has been used to detect many low-frequency earthquakes; the resulting catalogs of earthquakes provide a precise view of spatial and temporal evolution. Detailed analysis of the low-frequency earthquake activity has improved understanding of wider geophysical activity such as the occurrence of slow slip events and the migration of volcanic fluids.

Although matched filter method can be applied to data from only one station (for example, when the seismic network is small) (e.g. Vuan et al., 2018; Wech et al., 2020), the sum of CC between template waveforms and in the three components data measured at multiple observation stations was often used in most cases (e.g. Gibbons and Ringdal, 2006; Shelly et al., 2007). The analysis of low-frequency earthquakes usually employs a time window of $4-5 \mathrm{~s}$ for filtered waveforms at $1-4 \mathrm{~Hz}$ or $2-8 \mathrm{~Hz}$. The similarities of waveforms measured at multiple stations are evaluated by $\mathrm{CC}$ stacked over a seismic network. Earthquakes are identified by the summed CC being larger than a threshold value. Using stacked CC from multiple stations is advantageous over using of a single station to detect small-magnitude earthquakes including low-frequency earthquakes, because the $\mathrm{CC}$ from a single station always become high, as noise shares the same frequency band as the signal, resulting in substantial false detections. In other words, conventional matched filter technique is applicable only in regions sufficiently covered by observation stations and with well-determined seismic catalogs. Single-station matched filter method could potentially be effective in various regions in which microearthquakes occur, thus allowing detection of much smaller events that are only recorded by a single station (e.g. Vuan et al., 2018). However, much improvements of 
technique is required to maintain the quality of the catalog.

As CC can be calculated quickly, they can be used easily to evaluate waveform similarity. However, they do not necessarily evaluate the overall similarity because CC are generally sensitive to a portion of the waveform depending on their calculation formula. To reduce the contribution of large-amplitude parts, Gao and Kao (2020) proposed a method of dividing the time window, which could effectively distinguish seismic waves generated from different epicenters. The present work, unlike the previous study, concerns the detection of low-frequency earthquakes, so we cannot expect to improve detection accuracy by dividing the time window, due to the sharing of the same frequency band between signal and noises. Therefore, we tried to effect improvements by introducing another index in addition to $\mathrm{CC}$.

Statistical studies have proposed using other indices in addition to $\mathrm{CC}$ such as the mutual information (MI), the maximum information coefficient (Reshef et al., 2011), and the total information coefficient (Reshef et al., 2016). These indices show the similarity of two data sets, including non-linear relationships not evaluated by CC. MI has been used to evaluate electron correlation in the fields of chemical physics (Sagar and Guevara 2005) and medical imaging (Pluim et al. 2003). Various studies have used other indices, but they are generally more computationally costly than MI. Therefore, we introduce MI, which can evaluate with low computational cost the degree of waveform similarity in small-amplitude parts. In order to take advantage of both MI and CC, we propose a new method for detecting deep low-frequency (DLF) earthquakes using their product (called MICC) as an index.

\section{Data and Method}

This study considers the waveforms of DLF earthquakes in Kirishima volcano, one of the most active volcanoes in Japan. Waveform data are from the high-sensitivity seismograph network (Hi-net) of NIED (Okada et al. 2004; National Research Institute for Earth Science and Disaster Resilience 2019). We apply a band-pass filter of 1-8 Hz, and decimate the waveform from 100 to $25 \mathrm{~Hz}$ sampling before calculations.

Conventional matched filter usually evaluates detection using stacked CC between observed and template waveforms of three components measured at multiple seismic stations. The template is selected from previously observed earthquakes. The template events used here are DLF earthquakes in the unified catalog of the Japan 
112 Metrological Agency (JMA) (e.g. Katsumata and Kamaya, 2003). The CC of

113 component $j$ at seismic station $i$ are calculated as

$$
\operatorname{CC}\left(i, j, t_{\mathrm{tg}}+\Delta t_{i}\right)=\frac{\sum_{\tau}\left(v_{\mathrm{tp}}\left(i, j, t_{\mathrm{tp}}+\Delta t_{i}+\tau\right) v_{\mathrm{tg}}\left(i, j, t_{\mathrm{tg}}+\Delta t_{i}+\tau\right)\right)}{\sqrt{\sum_{\tau}\left(v_{\mathrm{tp}}{ }^{2}\left(i, j, t_{\mathrm{tp}}+\Delta t_{i}+\tau\right)\right)} \sqrt{\sum_{\tau}\left(v_{\mathrm{tg}}{ }^{2}\left(t_{\mathrm{tg}}+\Delta t_{i}+\tau\right)\right)}}(1)
$$

115 Here, $v_{\mathrm{tp}}(i, j, t)$ and $v_{\mathrm{tg}}(i, j, t)$ are the velocities of the template earthquake and target data

116 at time $t$ of component $j$ in station $i$, respectively; $t_{\mathrm{tp}}$ and $t_{\mathrm{tg}}$ are the times of occurrence

117 of the template earthquake and the target event, respectively; $\Delta t_{i}$ is the time of S-wave

118 propagation from the origin of the template earthquake to its arrival at station $i ; \tau$

119 corresponds to each time step in the window length. This analysis sets the length of the

120 time window to be $8 \mathrm{~s}$, and allocates its center to the arrival time of the template S-wave

121 earthquake observed by the JMA. When the JMA did not determine the arrival time at a

122 station, we estimate it using the occurrence time in the JMA catalog and the JMA's 1D

123 velocity structure model (Ueno et al. 2002). When using multiple stations, CC are

124 summed as follows:

$$
\text { Summed } C C\left(t_{t g}+\Delta t_{i}\right)=\sum_{i} \sum_{j} C C\left(i, j, t_{t g}+\Delta t_{i}\right)
$$

126 This sum is high when the peak CC values in the waveforms of each station and each

127 direction are stacked. In other words, when the hypocenter of the target event is near that

128 of the template event, the stacked peak values make the summed CC high.

129 MI is defined using the normalized amplitudes of two variables. First, we 130 normalized the waveforms within each time window using the maximum absolute 131 amplitudes. We then assigned the normalized velocities of each time step in each time 132 window, $\bar{v}_{t p}(t)$ and $\bar{v}_{t g}(t)$, to the $\mathrm{x}$ - and y-axis respectively, as shown in Figure 1 . By 133 dividing the normalized velocities to $5 \times 5$ cells, we converted the velocities into integers $134 n_{\mathrm{tp}}$ and $n_{\mathrm{tg}}$ between 1 and 5 as follows:

$$
\bar{v}_{\mathrm{tp}}(t)=\frac{v_{\mathrm{tp}}(t)}{\max \left(\left|v_{\mathrm{tp}}(t)\right|\right)} \cdots(3)
$$

$$
\bar{v}_{\mathrm{tg}}(t)=\frac{v_{\mathrm{tg}}(t)}{\max \left(\left|v_{\mathrm{tg}}(t)\right|\right)} \cdots(4)
$$

$$
n_{\mathrm{tp}}(t)=\text { floor }\left(\left(\bar{v}_{\mathrm{tp}}(t)+1.4\right) * 2.5\right)\left(\text { When } \bar{v}_{\mathrm{tp}}(t)<1\right) \cdots(5)
$$




$$
n_{\mathrm{tp}}(t)=5 \quad\left(\text { When } \bar{v}_{\mathrm{tp}}(t)=1\right) \quad \cdots(7)
$$

$$
n_{t g}(t)=5 \quad\left(\text { When } \bar{v}_{t g}(t)=1\right) \quad \cdots(8)
$$

142 function converting a real number to the largest integer smaller than itself. The constants

1431.4 and 2.5 convert the velocity into integers. This calculation means that $\mathrm{n}=1$ when the 144 normalized velocity $\bar{v}(t)$ ranges from -1.0 to $-0.6, n=2$ when $\bar{v}(t)$ is between -0.6 145 and $-0.2, \mathrm{n}=3$ when $\bar{v}(\mathrm{t})$ is between -0.2 and $0.2, \mathrm{n}=4$ when $\bar{v}(\mathrm{t})$ is between 0.2 and 1460.6 , and $\mathrm{n}=5$ when $\overline{\mathrm{v}}(\mathrm{t})$ is between 0.6 and 1.0 .

147 Using the above integers, the MI is calculated as

$$
\operatorname{MI}(t)=\sum_{n_{\mathrm{tp}}=1}^{5} \sum_{n_{\mathrm{tg}}=1}^{5} p\left(n_{\mathrm{tp}}, n_{\mathrm{tg}}\right) \log \frac{p\left(n_{\mathrm{tp}}, n_{\mathrm{tg}}\right)}{p\left(n_{\mathrm{tp}}\right) p\left(n_{\mathrm{tg}}\right)}
$$

where $p\left(n_{t p}\right)$ and $p\left(n_{t g}\right)$ are the probabilities of $n_{\mathrm{tp}}$ and $n_{\mathrm{tg}}$, respectively; $p\left(n_{t p}, n_{t g}\right)$ is the joint probability in the cells of $n_{\mathrm{tp}}$ and $n_{\mathrm{tg}}$. As the time window is $8 \mathrm{~s}$ and the sampling rate is $25 \mathrm{~Hz}$ (i.e., 200 time-steps for each time window), we obtain $p\left(n_{t p}\right)$ and $p\left(n_{t g}\right)$ by dividing the number of points in the bins by 200 . use the sum of the information entropy $h_{\mathrm{tp}}$ and $h_{\mathrm{tg}}$ to normalize the MI using the following equations (cf. Zhang, 2015):

$$
\begin{aligned}
& h_{\mathrm{tp}}=\sum-p\left(n_{\mathrm{tp}}\right) \log p\left(n_{\mathrm{tp}}\right) \cdots(10) \\
& h_{\mathrm{tg}}=\sum-p\left(n_{\mathrm{tg}}\right) \log p\left(n_{\mathrm{tg}}\right) \cdots(11) \\
& \overline{\mathrm{MI}}=\frac{2.0 * \mathrm{MI}}{h_{\mathrm{tp}}+h_{\mathrm{tg}}} \quad \cdots(12) .
\end{aligned}
$$

159 Hereafter, we simply describe $\overline{\mathrm{MI}}$ as MI.

MI is an index that reflects the linear or non-linear relation between two variables. If the relation is linear, both MI and CC are high (Figure 1a). While if the relation is non-linear, MI has a non-zero value (Figure 1b). According to equation (1), CC 
163 gives more weight to data points with high amplitudes in phase, while MI increases when

164 the data points are concentrated in a small number of cells. In other words, CC are high 165 when the peaks of two variables match, even if the shapes of the waveforms are different 166 (Figure 1c).

167


168
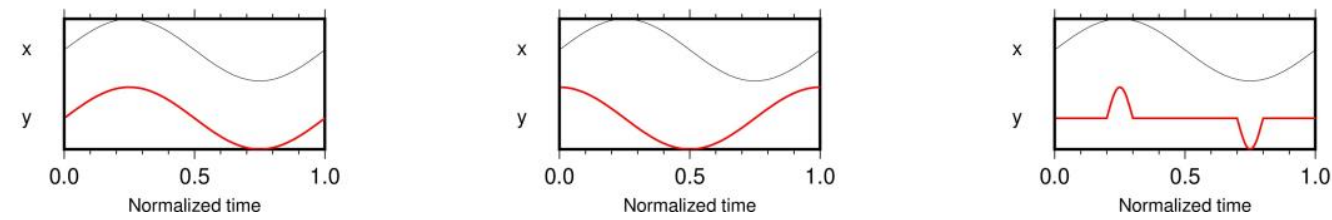

Figure 1 Scatter plots of relations between two variables (top) and their waveforms at the same time (bottom). MI and CC are given above the plots. Black grid lines correspond to the borders of the bins used to calculate MI. (a) Two variables with the same sinusoidal waves. (b) Two variables with sinusoidal waves and a time shift. (c) One variable with a sinusoidal wave and one with a spike-like wave. $\mathrm{CC}$ :

$$
\operatorname{MICC}(t)=\operatorname{MI}(t) \operatorname{CC}(t) \quad \cdots(13)
$$

178 The product of two variables contains the characteristics of MI, including information

179 about the small-amplitude parts, and the characteristics of the $\mathrm{CC}$, which correspond to

180 the consistency of large-amplitudes parts.

\section{Synthetic Tests}

183 We test how the index values of MI, CC, and MICC change in response to a variety 184 of noise and signals for DLF earthquakes. The analysis uses a synthetic data set 185 comprising two types of noise (Gaussian and sinusoidal) added to a template waveform 186 of data from a DLF earthquake recorded by the N.SUKH station at 22:03:56 (JST) on 7 
(a)

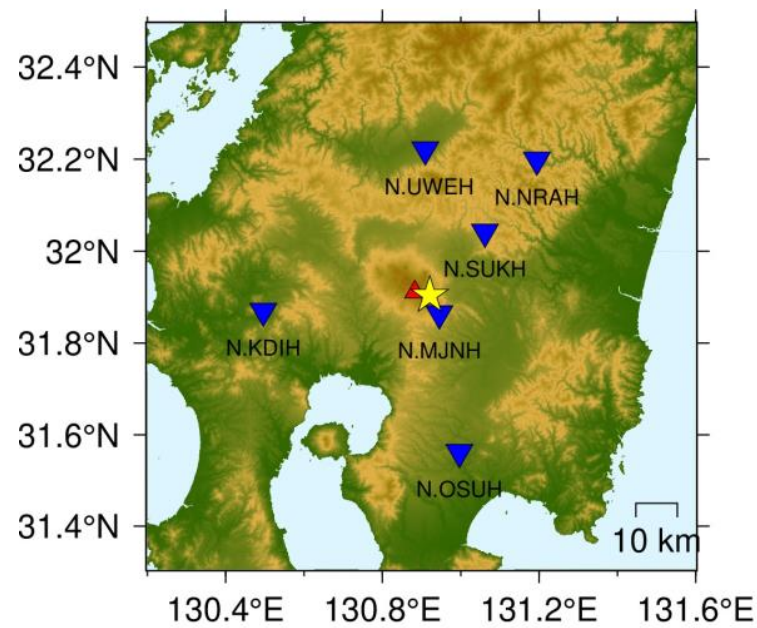

(b)

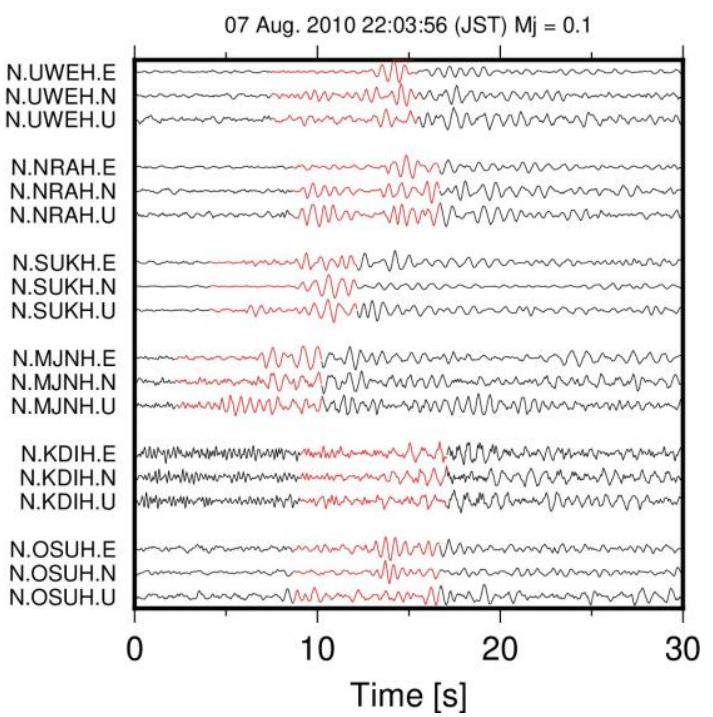

Figure 2 (a) Distribution of Hi-net stations (blue triangles) and the epicenter (yellow star) of the DLF earthquake (7 August 2010, 22:03:56, JST) used as a template here. The red triangle shows the location of Kirishima volcano. (b) Waveforms of three components of the earthquake recorded at the six stations. The horizontal axis is the time elapsed from the origin. Red parts of the waveforms show the template waveform used in this study.

\section{Gaussian noise}

We computed MI, CC, and MICC for the synthetic data. Artificial observation data were made by adding a filtered template waveform $(1-8 \mathrm{~Hz})$ with various amplitudes proportional to the signal-to-noise (SN) ratio at the N.SUKH station to Gaussian noise with a variance of 1 . The variance of the template waveform is the same as the value of the $\mathrm{SN}$ ratio.

When the SN ratio is small, the peaks of each index are unclear; in other words, DLF events are not detectable in the synthetic data (Figure 3a). When the SN ratio is high, $\mathrm{MI}, \mathrm{CC}$, and $\mathrm{MICC}$ have distinct peaks detectable by setting an appropriate threshold for each index (Figure 3b, c). In the case of Gaussian noise, DLF events are detectable using any of the indices when the SN ratio is over 0.5. 
(a) $\mathrm{SN}=0.2$

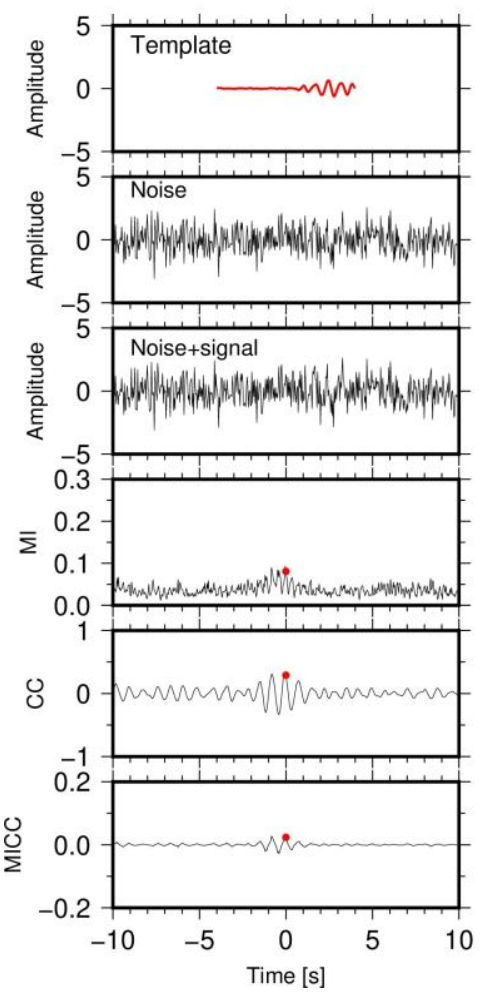

(b) $\mathrm{SN}=0.5$

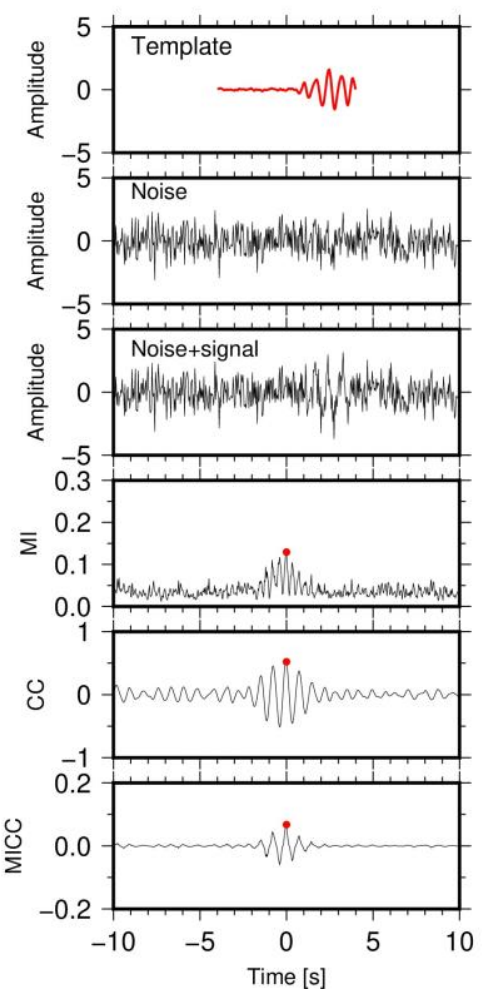

(c) $\mathrm{SN}=1.0$

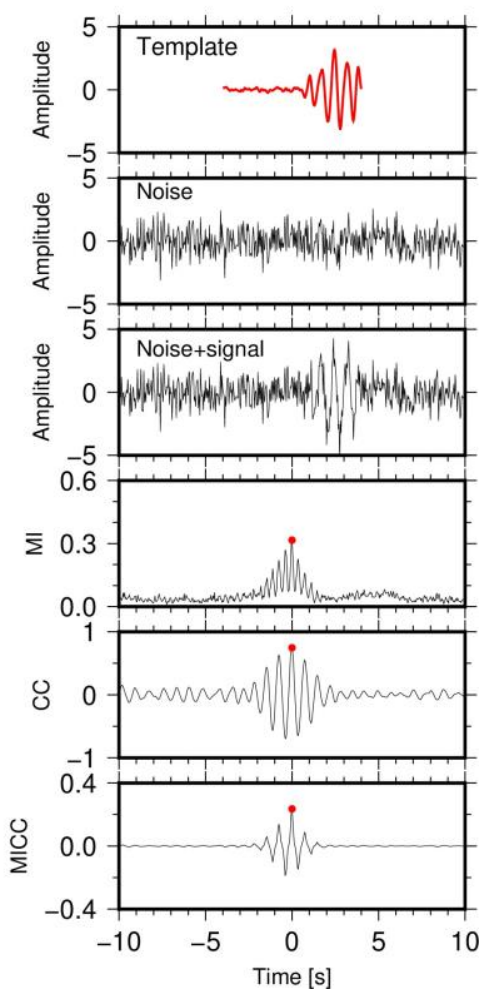

Figure $3 \mathrm{MI}, \mathrm{CC}$, and MICC for a signal comprising the template waveform with added Gaussian noise. The indices are calculated for each time step with the moving time window. The SN ratios are (a) 0.2, (b) 0.5, and (c) 1.0. Red dots show the index corresponding to the template signal.

The test results can verify how the three indices evaluate the similarity of the

215 waveforms. Scatter plots compare the amplitude of the template earthquake with that of

216 the target data (Figure 4a, b). The relationship becomes linear at high SN ratio (Figure

217 4b). CC rapidly increase when the $\mathrm{SN}$ ratio is relatively low, and reach a steady value as

218 the SN ratio becomes large; in contrast, MI gradually increases as the SN ratio rises

219 from 0 to 5 (Figure 4c). MICC gradually increases in any range of SN ratio

220 corresponding to an increase of MI and CC. 



(c)

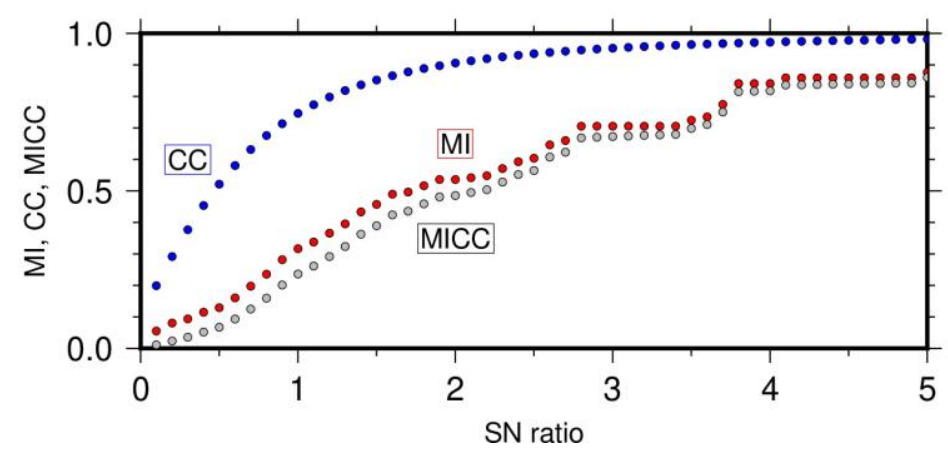

Figure 4 (a)-(b) Scatter plots of detection using a waveform comprising a

DLF earthquake signal with Gaussian noise at SN ratios of (a) 0.2 and (b) 1.0. Scatter plots (top panels) compare the amplitude of the template waveform with the amplitude of the target data at each time step. Bottom panels show the waveforms of the target data and template waveform at that time. (c) The three indices (MI, CC, and MICC) plotted with respect to the SN ratio of the waveform.

232 often overlap with the $1-8 \mathrm{~Hz}$ characteristic band of actual DLF earthquakes. The next

233 tested case has sinusoidal noise waves roughly matching the frequency band of DLF 234 earthquakes. 


\section{Sinusoidal noise}

As in the case of Gaussian noise, the test considers a template waveform with

237 added noise, this time a sinusoidal wave of $1.25 \mathrm{~Hz}$ frequency. The variance of the noise

238 is 1 . The variance of $\mathrm{CC}$ tends to be always high due to the phase similarity with the

239 noise (Figure 5). On the other hand, MI is small when the waveform does not include

240 the template signal, and a distinct peak can be observed compared with the CC.

241 Although CC is relatively large when the noise phases match with those of the template

242 event, the MI remains smaller than CC (Figures 6, 7). MICC has the most-distinct peak

243 corresponding to the signal, and allows the detection of DLF earthquakes at low SN

244 ratio. We therefore suggest that MICC as a proper and sensitive index to detect DLF 245 events.

(a) $\mathrm{SN}=0.2$

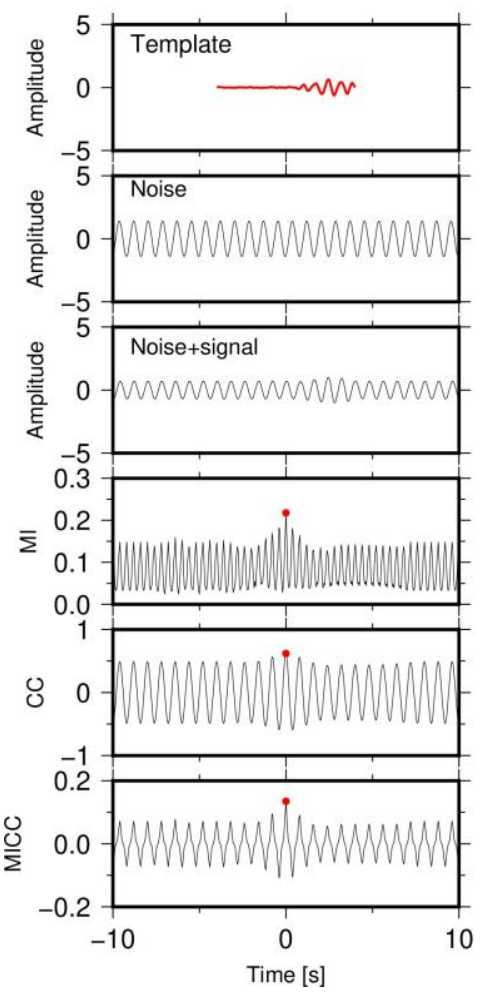

(b) $\mathrm{SN}=0.5$



(c) $\mathrm{SN}=1.0$



Figure $51.25 \mathrm{~Hz}$ sine wave used as noise combined with template waveforms at $\mathrm{SN}$ ratios of (a) 1.0, (b) 2.0, and (c) 3.0. The values of MI, CC, and MICC are calculated at each time. Red dots show the index corresponding to the template signal. 

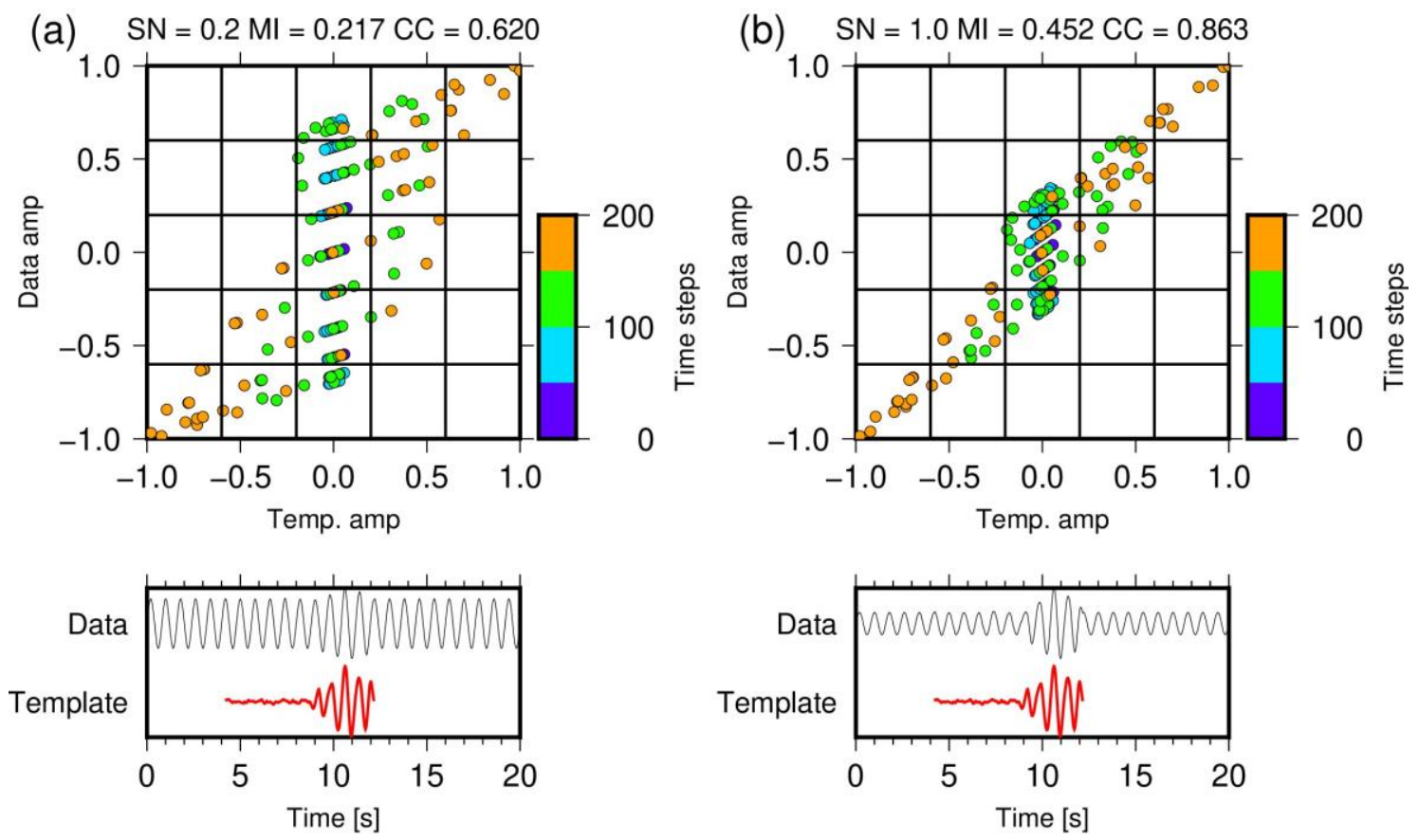

(c)



Figure 6 Scatter plots and relationship between the SN ratio of the waveform and 255 the MI, CC, and MICC for the synthetic waveform with sinusoidal noise. The figures are 256 as in Figure 4, but here the noise is a sinusoidal wave of frequency $1.25 \mathrm{~Hz}$. 

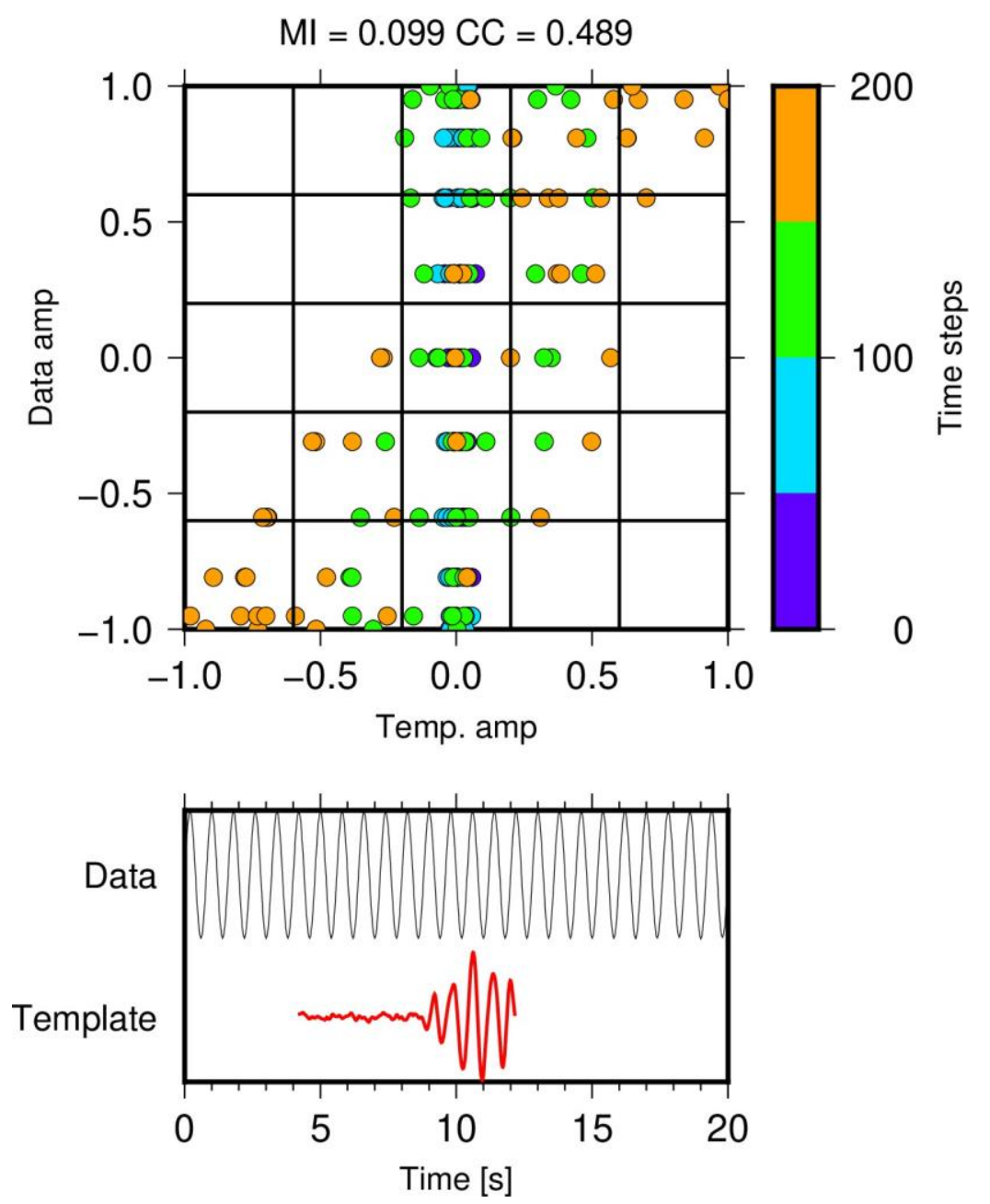

Figure 7 Scatter plot showing the correspondence between the sinusoidal wave noise and the template waveform, using the data from $12 \mathrm{~s}$ before Figures 5 and 6 . 


\section{Application to field data from DLF earthquakes at Kirishima volcano}

Here we apply the new method to field data. We selected 200 waveforms of DLF earthquakes as templates from the JMA catalog from 2004 to 2015 and tested continuous data observed in December 2010 at Kirishima volcano. Only two DLF earthquakes were observed in the catalog of JMA in the month. The waveform data are band-pass filtered at 1-8 Hz. For detection using a single station, we focus on threecomponent seismograms retrieved at the N.SUKH station (see Figure 2a), which has the highest SN ratio for DLF earthquakes at Kirishima volcano.

First, to determine the threshold value, we examine histograms of MI, CC, and MICC obtained from data for 21 December 2010, when intense DLF earthquakes occurred. An earthquake is counted as occurring when the index value is larger than the threshold. The time series of MI, CC, and MICC vibrate in the same frequency bands of the template waveform. The distribution of maximum values for each time series (Figure 8) is found by simply extracting the value larger than the values occurring both before and after. The distribution of each index differed among the three templates. Conventional matched filter based on summed CC often has the threshold value determined based on the median absolute deviation (e.g., Shelly et al., 2007, Peng and Zhao, 2009). However, it cannot be used for MI because MI is always positive and the shape of the distribution is different from the approximately Gaussian distribution of CC. We set here the threshold to be 0.35 for MICC, based on visual inspection of waveforms and the histogram distributions of the maximum value of each index (Figure 8). The thresholds for comparative analysis using only MI and CC are 0.45 and 0.85 , respectively. A DLF earthquake is detected when the index of any of the three components exceeds the threshold. Some earthquakes are detectable more than once by the different template earthquakes. To prevent double counting of any earthquake, we select only one event with the highest index in any $10 \mathrm{~s}$ time window and neglect the other events. After compiling the detection catalogs, results corresponding to the daily artificial signal at 9:00:00 (JST) in the Hi-net data are removed. 



Figure 8 Histograms of the maxima of three indices (MI, CC, and MICC) extracted from each time series. Vertical axes correspond to the number of samples in each bin

297 (width, 0.01). The time at the top of each panel is the time of occurrence of the template

298 event (JST). Applied data are for one day, 21 December 2010. Red lines show the

299 threshold value for each index.

Figure 9 gives the calculated time variations of MI, CC, and MICC for each time step with a sampling rate of $25 \mathrm{~Hz}$. MICC has very distinct detection peaks, while CC always vibrates with a large variance. In this data set, the summed $\mathrm{CC}$ in the three components of six stations does not show distinct peaks corresponding to some DLF earthquakes detected using the proposed single-station method. For example, the events at $05: 48$ on December 21 were detected in the single-station method, however, summed $\mathrm{CC}$ does not have the peak corresponding to the events. Then, there are some cases in which no peaks are found in the summed $\mathrm{CC}$ because the amplitude is small or the hypocentral location is slightly different from those of the template event, suggesting that

310 the single-station method is suitable for comprehensive detection. The 311 comprehensiveness of the method can be also seen from the fact that the peaks of MICC 
312 correspond to large amplitudes in the velocity waveform except some parts corresponding

313 to the signals of earthquakes occurring in other regions.

314 MICC identified 354 DLF earthquakes in the test month. Even using data from a

315 single station achieved a temporal change of the cumulative number of DLF events

316 similar to that observed from the catalog of events compiled from six stations' data

317 (Figure 10). The catalogs include step-like increases in the number of DLF earthquakes

318 on December 5 and December 12, and the difference between the catalogs over the

319 month is small. This trend is generally consistent across the results from any of the three

320 indices. As many of the earthquakes detected by these indices have very small SN

321 ratios, it is not possible to distinguish between a true signal and a false detection even

322 by visual inspection of the waveforms. Although the MICC peak is the most distinct and

323 the detection looks good, the accuracies of the detection of the three single-station

324 indices are quantitatively evaluated in discussion. 



Figure 9 Values of MI, CC, and MICC from 0:00 to 12:00 on December 21,

328 2010. The lowest panel shows the velocity waveform. The template earthquake is the

329 earthquake shown in Figure 1. Red lines show the threshold of detection. Green

330 triangles in each panel show the detected events by the template using the index. White 
331 triangles show the detection using other templates.

332

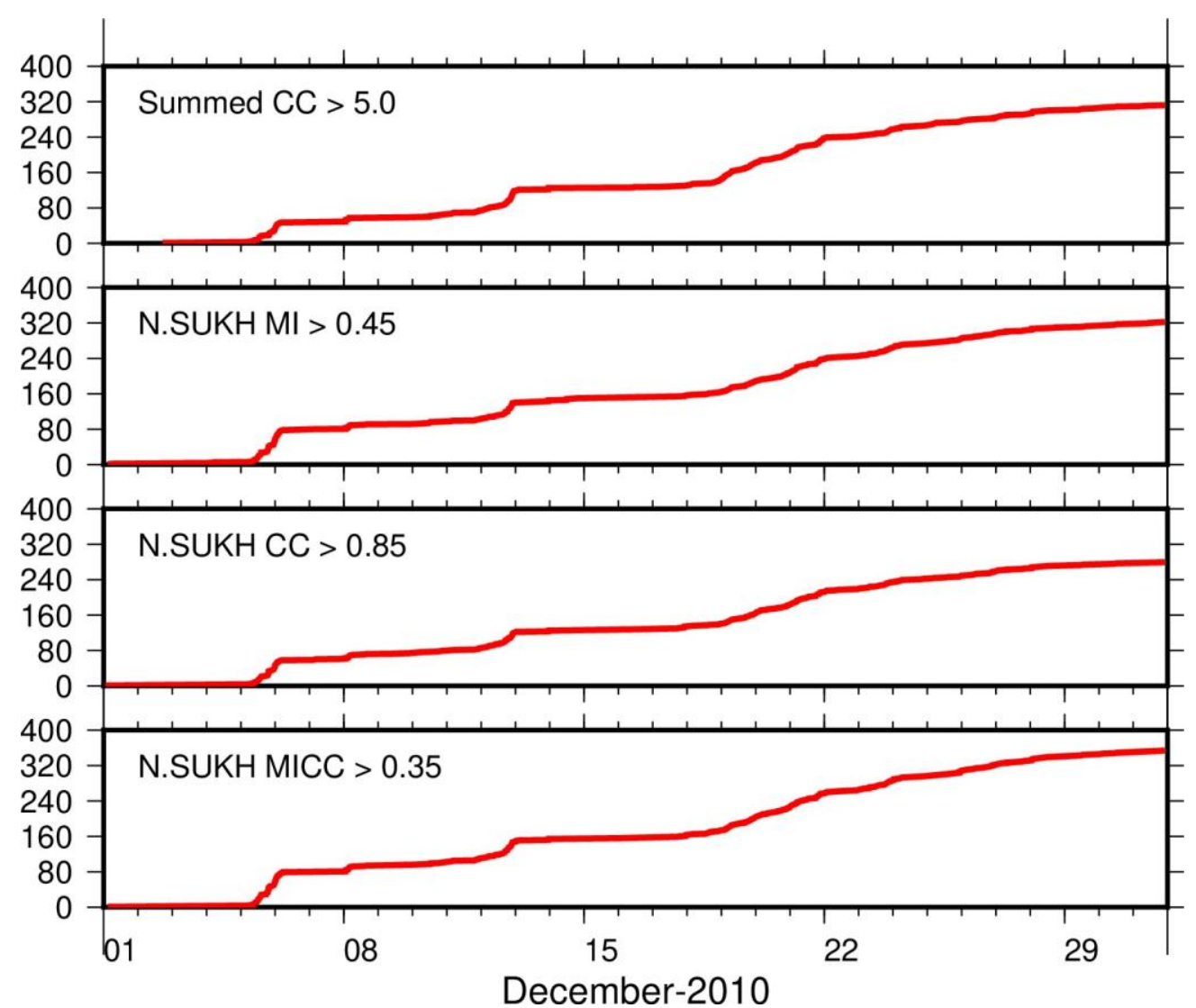

Figure 10 Cumulative number of detected DLF earthquakes based on each index

335 during December 2010. From the top, the indices are summed CC of the six stations 336 and MI, CC, and MICC of the N.SUKH station. 


\section{Discussion}

To confirm which index performs best, we compared the catalog obtained using each of three indices with one based on conventional matched filter method using 341 summed CC (e.g. Gibbons and Ringdal, 2006; Kato and Nakagawa, 2020; Kurihara et al., 342 2019; Shapiro et al., 2017; Shelly et al., 2007; Yukutake et al., 2019). Using a threshold 343 of 5.0 for conventional detection using the summed $\mathrm{CC}$ of three components of the six 344 seismic stations detected 312 events throughout December 2010. We assume that the 345 conventional method provides a true catalog against which the performance of other 346 methods can be compared using the threat score (also called the critical success index), 347 which is often used in the weather forecasting (Japan Meteorological Agency 2019). The score is defined as follows:

$$
\text { Threat score }=\frac{T P}{T P+F P+F N} \quad \cdots(14) \text {, }
$$

350 where TP, FP, and FN are the numbers of true positive, false positive, and false negative events, respectively. TP events are those detected in the catalogs from both the conventional multiple-station method and the single-station method. FP events are those detected by the single-station method but not the conventional method. FN events are those detected conventionally but not by the single-station method. The threat score is 0.366 for MI, 0.261 for CC, and 0.441 for MICC applied to single-station data. As MICC gives the highest threat score, it appears to give the best performance among the three indices.

Next assessed is the effect of changing the detection threshold for each catalog.

359 The threat score is maximized at 0.393 for the MI catalog using a threshold of 0.430 , at 3600.354 for CC with a threshold of 0.800 , and at 0.441 for MICC with a threshold of 0.350

361 (Figure 11). The threat score for the MICC catalog remains similar within a broad range 362 of threshold values of 0.3 to 0.4 , indicating that the same accuracy can be obtained 363 regardless of which threshold is selected within this range. 



Figure 11 The threat score and the number of TP, FP, and FN events.

The upper panels show the threat score for three indices. Horizontal axis corresponding to the threshold values. Red, blue, and green circles in the lower panels show the number of TP, FN, and FP events for each threshold value.

We compare the maximum value of summed $\mathrm{CC}$ in the $5 \mathrm{~s}$ before and after the time with that of each index at the single station (Figure 12). The summed CC is positively correlated with each of MI, CC, and MICC applied to a single station's data. Especially, MICC shows the best positive relationship with the summed CC.
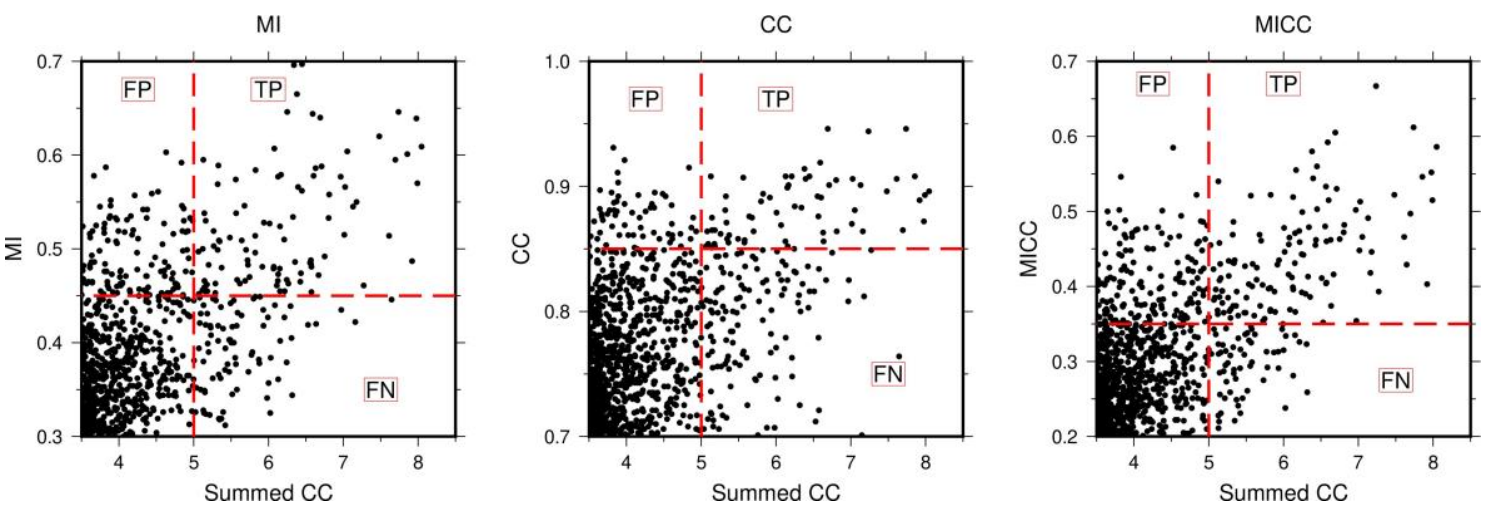

Figure 12 Relations between summed CC (horizontal axis) and three indices (vertical axis) of a component of the data from N.SUKH station. Black dots correspond to each event. Red broken lines show the threshold of detection.

The threat score for the MICC catalog is the highest among the three catalogs; however, the value seems low. We next assess the quality of the catalog compiled using MICC. Of the 88 events detected with summed CC over 6.0, 80 are also included in the MICC catalog. On the other hand, of the 354 events detected using MICC, $85 \%$ of the events (302 events) are also in the catalog of summed CC with the threshold of 4.0. In other words, the FP and FN events included those which are around the threshold value, 
and the threat score of 0.441 does not mean that only $44.1 \%$ of DLF earthquakes are detected. The single-station catalog probably includes DLF earthquakes with very small magnitudes that are only observed at the single station (N.SUKH); however, this comparison cannot distinguish FP detections from actual small DLF earthquakes.

This study calculates MI by dividing the points of velocity-seismograms into $5 \times 5$ cells. The number of divisions was determined by evaluating the clarity of the peaks corresponding to the signal. Comparing calculations for each number of divisions for the continuous waveform showed that odd-numbered divisions such as $3 \times 3$ and $5 \times 5$ give sharper peaks than even-numbered division such as $4 \times 4$ when the number of divisions is small (Figure 13). This is because the points near the origin in even-numbered divisions. belong to different cells due to slight differences of the seismograms. This leads to a decrease in the MI when considering the distribution diagram as shown in Figure 4. As $3 \times 3$ division has a large variance for parts not including signals of DLF earthquakes, $5 \times 5$ is considered suitable for detection in the three data sets. On the other hand, as the number of divisions is increased, the baseline of MI rises and the peaks become generally less sharp (Figure 13) because there are too many cells relative to the number of the points. Therefore, for our dataset with $25 \mathrm{~Hz}$ sampling and an $8 \mathrm{~s}$ time window, $5 \times 5$ division is optimal.

In this study, we calculate MI by dividing the points of velocity-seismograms into the

$5 \times 5$ cells. The number of divisions was determined by evaluating the clarity of the peaks corresponding to the signal. As a result of the calculation for each number of divisions for the continuous waveform, the peak of the odd-numbered division such as $3 \times 3$ and 5 $\times 5$ is sharper than that of the even-numbered division such as $4 \times 4$ when the number of divisions is small (Figure 13). This is because the points near the origin belong to different cells due to slight differences of the seismograms. It leads to a decrease in the MI when considering the distribution diagram as shown in Figure 4. In addition, $3 \times 3$ has a large

412 variance for the parts which do not include signals of DLF earthquakes, therefore, $5 \times 5$

413 is suitable for the detection in the three data sets. On the other hand, as the number of 414 divisions is increased, the baseline of MI rises and the peaks become duller in general 415 (Figure 13) because the number of cells is too much compared to the number of the points. 416 Therefore, for our dataset with $25 \mathrm{~Hz}$ sampling and an 8-second time window, we 417 determined that a $5 \times 5$ split was optimal. 
Using MICC with a single station's data is a potentially powerful tool,

419 especially for monitoring shallow volcanic earthquakes occurring beneath the crater

420 because there cannot be many seismic stations in that region due to the risk and low

421 accessibility. In addition, the single-station method will improve the completeness of

422 small-magnitude volcanic seismicity studies, deepening understanding about volcanic

423 activity beneath the crater. As in previous cases of single-station analysis (Vuan et al.

424 2018; Wech et al. 2020), this method will also contribute to the monitoring of other low-

425 SN ratio earthquakes, such as DLF earthquakes and swarm earthquakes that can be 426 observed only with part of an observation network.

2010-12-21 00:00:00 N.SUKH North-South

2010-12-21 00:00:00 N.SUKH North-South
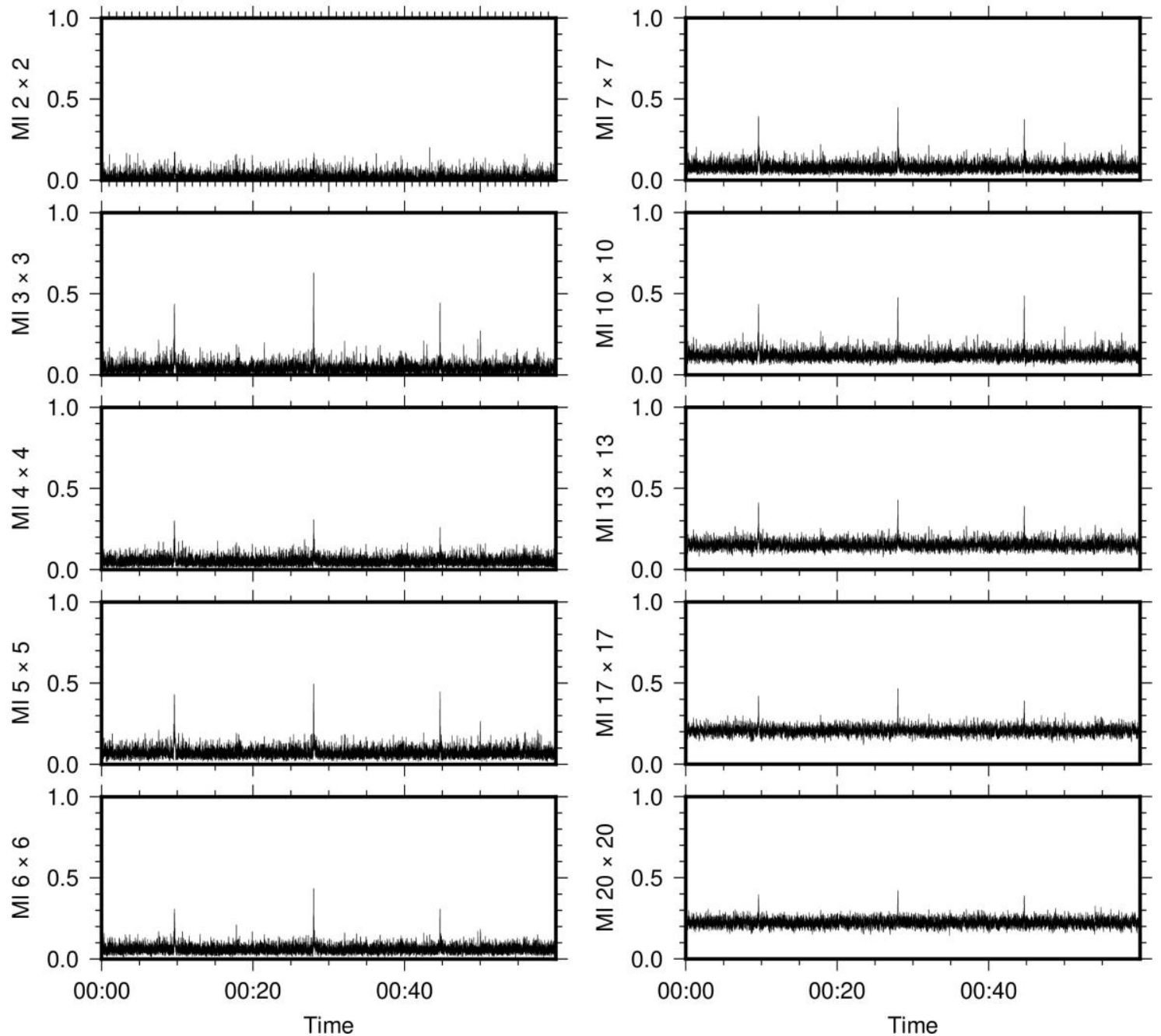

Figure 13 Time series of MI using 10 different divisions. The time window is one hour

429 from 00:00:00, 21 December 2010 (JST). The template earthquake and observation

$430 \quad$ station are as in Figure 9. 


\section{Conclusions}

433 This study developed a new matched filter method of earthquake detection using 434 MICC applied to a single station's data. Tests using synthetic waveforms revealed that 435 using MICC gave more-distinct peaks than MI or CC. Applying this method to DLF 436 earthquake data from Kirishima volcano in December 2010 detected 354 DLF 437 earthquakes. Comparison with conventional matched filter applied to multiple stations' 438 data showed the large detection accuracy of the proposed method. Overall, the proposed 439 single-station matched filter technique could be useful in various regions where 440 observations from multiple stations are not possible, as it can detect microearthquakes 441 using only a small number of stations and templates.

\section{Declarations}

\section{Ethics approval and consent to participate}

$445 \quad$ Not applicable

\section{Consent for publication}

$447 \quad$ Not applicable

448

449 List of abbreviations

450

451 MFT: Matched filter technique

452 DLF: Deep Low-Frequency

453 CC: Correlation coefficients

454 MI: Mutual information

455 MICC: Product of mutual information and correlation coefficients

456 Hi-net: High-sensitivity seismograph network

457 NIED: National Research Institute for Earth Science and Disaster Resilience

458 JMA: Japan meteorological agency

459 MAD: Median absolute deviation

460 TP: True positive

461 FP: False positive 
FN: False negative

\section{Availability of data and materials}

We used the Hi-net seismic observation data from NIED (National Research Institute for Earth Science and Disaster Resilience 2019) and JMA's unified earthquake catalog. Those are available from the web page of NIED Hi-net (http://www.hinet.bosai.go.jp).

\section{Competing interests}

474

The authors declare that they have no competing interests.

475

476

477

\section{Funding}

478 This work was supported by Japan Science and Technology Agency (JST) CREST

479 Grant Number JPMJCR1763, Japan.

480

\section{Authors' contributions}

RK mainly design and analyze the data and write the paper. AK advises and discusses the contents of this paper. HN and SK advise about indices in statics and discuss the paper.

\section{Acknowledgments}

487 We used Generic Mapping Tools for drawing figures (Wessel and Smith 1998) and 488 collated the Hi-net seismic observation data (http://www.hinet.bosai.go.jp) from NIED 489 (National Research Institute for Earth Science and Disaster Resilience 2019). We used 490 JMA's unified earthquake catalog (http://www.jma.go.jp) and the computer systems of 491 the Earthquake and Volcano Information Center of the Earthquake Research Institute, 492 the University of Tokyo. This work was supported by the JST CREST (grant number 493 JPMJCR1763) and partially supported by the Ministry of Education, Culture, Sports, 494 Science and Technology (MEXT) of Japan, under its The Second Earthquake and 
Volcano Hazards Observation and Research Program (Earthquake and Volcano Hazard

496 Reduction Research).

497

498

499

\section{References}

Gao D, Kao H (2020) Optimization of the match-filtering method for robust repeating earthquake detection: The multi segment cross-correlation approach. J Geophys Res: Solid Earth 125(7):1-19. https://doi.org/10.1029/2020JB019714

Gibbons SJ, Ringdal F (2006) The detection of low magnitude seismic events using array-based waveform correlation. Geophys J Int 165(1):149-166.

https://doi.org/10.1111/j.1365-246X.2006.02865.x

Japan Meteorological Agency (2019) Outline of the operational numerical weather prediction of the Japan Meteorological Agency. Japan Meteorological Agency.

Retrieved from http://www.jma.go.jp/jma/jma-eng/jma-center/nwp/outline2013nwp/pdf/outline2013_03.pdf

Kato A, Nakagawa S (2020) Detection of deep low-frequency earthquakes in the Nankai subduction zone over 11 years using a matched filter technique. Earth Planets Space 72(1):128. https://doi.org/10.1186/s40623-020-01257-4

Katsumata A, Kamaya N (2003) Low-frequency continuous tremor around the Moho discontinuity away from volcanoes in the southwest Japan. Geophys Res Lett 30(1):20-1-20-4. https://doi.org/10.1029/2002GL015981

Kurihara R, Obara K, Takeo A, Tanaka Y (2019) Deep low-frequency earthquakes associated with the eruptions of Shinmoe-dake in Kirishima volcanoes. J Geophys Res: Solid Earth 124(12):13079-13095. https://doi.org/10.1029/2019JB018032

National Research Institute for Earth Science and Disaster Resilience (2019) NIED Hinet. National Research Institute for Earth Science and Disaster Resilience. https://doi.org/10.17598/nied.0003

Okada Y, Kasahara K, Hori S, Obara K, Sekiguchi S, Fujiwara H, Yamamoto A (2004) Recent progress of seismic observation networks in Japan — Hi-net, F-net, K-NET and KiK-net-. Earth Planets Space 56(8):xv-xxviii. https://doi.org/10.1186/BF03353076

Peng Z, Zhao P (2009) Migration of early aftershocks following the 2004 Parkfield earthquake. Nat Geosci 2:877-881. https://doi.org/10.1038/ngeo697

Pluim JPW, Maintz JBA, Viergever MA (2003) Mutual-information-based registration 
of medical images: a survey. IEEE Trans Med Imaging 22(8):986-1004. https://doi.org/10.1109/TMI.2003.815867

530 Reshef DN, Reshef YA, Finucane HK, Grossman SR, McVean G, Turnbaugh PJ, et al. (2011) Detecting novel associations in large data sets. Science 334(6062):15181524. https://doi.org/10.1126/science. 1205438

Reshef YA, Reshef DN, Finucane HK, Sabeti PC, Mitzenmacher M (2016) Measuring dependence powerfully and equitably. J Mach Learn Res 17:1-63.

Sagar RP, Guevara NL (2005) Mutual information and correlation measures in atomic systems. J Chem Phys 123(4):044108. https://doi.org/10.1063/1.1953327 Deep and shallow long-period volcanic seismicity linked by fluid-pressure transfer. Nat Geosci 10(6):442-445. https://doi.org/10.1038/ngeo2952

Shelly DR, Beroza GC, Ide S (2007) Non-volcanic tremor and low-frequency earthquake swarms. Nature (7133):305-307. https://doi.org/10.1038/nature05666

Ueno H, Hatakeyama S, Aketagawa T, Funasaki J, Hamada N (2002). Improvement of hypocenter determination procedures in the Japan Meteorolocy Agency. Quar J Seismol 65:123-131. Retrieved from https://www.jma.go.jp/jma/kishou/books/kenshin/vol65p123.pdf

Vuan A, Sugan M, Amati G, Kato A (2018) Improving the detection of low-magnitude seismicity preceding the Mw 6.3 L'Aquila Earthquake: Development of a scalable code based on the cross correlation of template earthquakes. Bull Seismol Soc Am 108(1):471-480. https://doi.org/10.1785/0120170106

Wech AG, Thelen WA, Thomas AM (2020) Deep long-period earthquakes generated by second boiling beneath Mauna Kea volcano. Science 368(6492):775-779. https://doi.org/10.1126/science.aba4798

Wessel P, Smith WHF (1998) New, improved version of generic mapping tools released. Eos, Trans Am Geophys Union 79(47):579-579. https://doi.org/10.1029/98EO00426

Yukutake Y, Abe Y, Doke R (2019) Deep low-frequency earthquakes beneath the Hakone Volcano, Central Japan, and their relation to volcanic activity. Geophys Res Lett 2019GL084357. https://doi.org/10.1029/2019GL084357

Zhang P (2015) Evaluating accuracy of community detection using the relative normalized mutual information. J Stat Mech: Theory Exp 2015(11):P11006. 
https://doi.org/10.1088/1742-5468/2015/11/P11006

562 


\section{Supplementary Files}

This is a list of supplementary files associated with this preprint. Click to download.

- graphicalabst.jpg 